\title{
Targeting Higher Levels of Tau Protein in Ukrainian Patients with Wilson's Disease
}

\author{
Yevgeniya Lekomtseva · Ivan Voloshyn-Gaponov · Gorbach Tatayna
}

Received: January 28, 2019 / Published online: March 27, 2019

(C) The Author(s) 2019

\begin{abstract}
Introduction: Wilson's disease (WD) is a rare genetic disorder of copper metabolism in which impaired copper homeostasis may enhance amyloid aggregation and trigger neurodegeneration. Tau protein is a highly soluble microtubule-associated phosphoprotein that plays a significant role in microtubule stabilization; it is also a critical component of neurotoxic degenerative mechanisms. Tau has been shown to be involved in neuronal degeneration and axonal damage, and impaired copper metabolism has been shown to be involved in copper
\end{abstract}

Enhanced digital features To view enhanced digital features for this article go to https://doi.org/10.6084/ m9.figshare.7857017.

Y. Lekomtseva $(\varangle) \cdot$ I. Voloshyn-Gaponov Department of Neurology, State Institute of Neurology, Psychiatry and Narcology of the National Academy of Medical Sciences of Ukraine, Academic Pavlov Street, 46, 61068 Kharkiv, Ukraine e-mail: yevgeniyalekomtseva@gmail.com

\section{Y. Lekomtseva}

Department of Functional Neurosurgery and Paroxysmal States, State Institute of Neurology, Psychiatry and Narcology of the National Academy of Medical Sciences of Ukraine, Academic Pavlov Str, 46, 61068 Kharkiv, Ukraine

\section{G. Tatayna}

Department of Biochemistry, Kharkiv National Medical University, Avenue of Science, 4, Kharkiv 61000, Ukraine intoxication and thus associated with the processes of neurodegeneration and cellular damage. We have therefore investigated tau protein as a potential marker of axonal impairment and neurodegeneration.

Methods: Patients with WD $(n=47 ;$ mean age \pm standard deviation [SD] $30.19 \pm 7.87$ years; mean disease duration : $10.06 \pm 3.9$ years) and healthy controls (HC; $n=30$; mean age $29.6 \pm 4.73$ years) were tested for serum tau protein levels using an enzymelinked immunosorbent assay method. All patients were receiving a stable penicillamine dose as ongoing therapy.

Results: Patients with WD had a higher mean tau protein level than did the HC $(221.7 \pm 135.1 \quad$ vs. $71.14 \pm 20.56 \mathrm{pg} / \mathrm{mL}$, $p<0.0001)$. Patients with WD also had abnormally high serum tau protein levels ( $t$ statistic $6.047,95 \%$ confidence interval -218.2 to -95.86) in both the cerebral and hepatocerebral forms of WD, with patients having the cerebral form showing a tendency toward higher tau levels. We found that tau protein did not differ according to gender, disease duration, age at disease onset, ceruloplasmin serum level and copper serum level.

Conclusion: This study provides novel data revealing that high tau protein levels in WD patients could be a potential biomarker for axonal impairment and possible neuronal damage due to tau protein, leading to neurodegeneration in WD. 
Keywords: Axonal damage; Neurodegeneration; Tau protein; Wilson's disease

\section{INTRODUCTION}

Wilson's disease (WD) is a rare autosomal recessive genetic disorder characterized by the accumulation of copper and subsequent copper toxicity predominantly in the brain, liver and other target organs due to mutations in the $A T P 7 B$ gene. The prevalence of $\mathrm{WD}$ in the general population of Europe is estimated at $1-2 / 60,000$, and that in Ukraine is considered to be about $1 / 45,000$ [1-3].

Tau protein is a novel class of axonally derived microtubule-associated proteins that contain many phosphorous groups and which appear to function in the formation and maintenance of axons by influencing of microtubule organization [4-7]. Heat-stable tau protein regulates and promotes microtubule assembly and stability. There are several molecular isoforms of tau protein, which can be distinguished by the number of binding domains. Tau plays an important role in the modification of tubulin monomers into microtubules, in the maintenance of the neuron and glial cytoskeleton and in axonal transport [8-11]. It is also a critical component in the neurotoxic degenerative mechanisms: for example, the aggregation of its specific units into filamentous inclusions is the most common feature of intraneuronal and glial-fibril lesions in neurodegenerative disorders such as Alzheimer disease's (AD) and tauopathies [5, 9, 11-13]. Recent studies have demonstrated that tau has the potential to be a diagnostic agent for detecting early axonal damage in patients with brain trauma [14, 15]. Other studies have demonstrated that tau-associated genes, proteins and tau phosphorylation are abnormal manifestations in patients with intractable epilepsy [16], multiple sclerosis (MS) [17-19] and stroke [20].

A number of studies have shown that copper dyshomeostasis may enhance the progression of neurodegeneration. Alterations in copper metabolism have been associated with increased metal-related oxidative stress and the formation and precipitation of $\beta$-amyloid
$[1,21]$. Impaired cellular copper homeostasis may trigger neurodegeneration through different mechanisms, such as the induction of oxidative stress $[22,23]$, promotion of $\alpha$-synuclein and glial-fibril aggregations [24-26] and activation of microglial cells [3, 27]. Mechanisms involving the accumulation of toxic substances, such as copper, may lead to widespread neurodegeneration as free copper itself is very toxic and can produce irreversible cellular damage [28, 29]. Impaired copper transition enhances amyloid aggregation and neurotoxicity, as shown in animal models where copper was found to also promote tau aggregation $[2,7,9]$. WD represents an interesting neurological problem for which the roles of these conditions are still unknown.

The identification of biomarkers of axonal damage is of great clinical significance. Consequently, we undertook a study to examine pathological tau protein in patients with WD. We hypothesized that because tau is involved in neuronal degeneration and axonal damage and because impaired copper metabolism with the resultant copper intoxication is associated with the processes of neurodegeneration and cellular damage, it is possible that serum tau protein level is a potential marker of axonal impairment and neurodegeneration in patients with WD versus controls.

\section{METHODS}

\section{Patients}

Forty-seven patients with WD (mean age \pm standard deviation [SD] $30.19 \pm 7.87$ years; mean disease duration $10.06 \pm 3.9$ years) and 30 age-matched healthy controls (HC; $29.6 \pm 4.73$ years, range $18-36$ years) were tested for serum tau protein levels at baseline. The HC had no known neurological and psychiatry diseases. The state of disease of all patients was sporadic in nature, and all patients were born in the Ukraine. At the time of the study, all WD patients were on a stable dose of penicillamine, with $21.27 \%$ of patients having been treated for at least 5 years. 
All patients provided written informed consent. All procedures performed in this study involving human participants were in accordance with local ethic committee and with the 1964 Helsinki declaration and its later amendments or comparable ethical standards.

\section{Procedures}

Microtubule-associated tau protein was quantified as follows. Aliquots of blood $(10.0 \mathrm{~mL})$ were collected in BD Vacutainer ${ }^{\circledR}$ collection tubes (Becton, Dickinson and Co., Franklin Lakes, NJ, USA; cat. reference 367896) and allowed to clot for $30 \mathrm{~min}$ at room temperature, following which the samples were centrifuged at 3000 $g$ for $15 \mathrm{~min}$ and the serum collected. Serum samples were then frozen at $-30-80{ }^{\circ} \mathrm{C}$ until the assay was performed. Tau concentrations in the samples were measured by an enzyme-linked immunosorbent assay (ELISA) method using a commercially available Human Tau ELISA kit (Sigma, St. Louis, MO, USA) according to the manufacturer's protocol. A Stat Fax ${ }^{\circledR}$ chemistry analyzer (Awareness Technology Inc., Palm City, FL, USA) at the Department of Clinical Biochemistry, Kharkiv National Medical University was used to make the measurements. The optical density was determined with a microplate reader set to $450 \mathrm{~nm}$; the serum total tau protein levels were presented as picograms per milliliter. The detection limit of the tau protein assay was $33 \mathrm{pg} / \mathrm{mL}$, which corresponds to the lowest standard on the calibration curve. All standards and samples collected from the $\mathrm{HC}$ and patients were measured in duplicate with a coefficient of variation of $<10 \%$. The protocols were approved by the Health Research Ethics Committee.

The ceruloplasmin level was determined by turbidimetry (model AU680; Olympus Corp., Tokyo, Japan) at $340 \mathrm{~nm}$, and the protein concentration was determined using an assay kit (Spinreact S.A., Girona, Spain; model N1102062) according to standard procedures.

\section{Statistical Analyses}

Data were analyzed according to their distribution. Age and disease duration were compared between groups (patients and HC) with the $\chi^{2}$ test; parametric tests were used for normally distributed data, and nonparametric tests were use for abnormally distributed data. Kruskal-Wallis and Mann-Whitney $U$ tests were applied in the Prism software package (GraphPad Software Inc., La Jolla, CA, USA). To determine differences between groups, multivariate analysis taking covariates into consideration was performed; univariate analysis was performed to assess the relationships between various factors. All reported $p$ values are two-tailed; $p$ values of $\leq 0.05$ were considered statistically significant.

\section{RESULTS}

\section{Patient Characteristics}

All 47 patients (24 men, 51.06\%; 23 women, 48.94\%) were aged between 19 and 54 years at entry into the study. Established WD was diagnosed using the scoring system developed at the 8th International Meeting on Wilson's Disease (2001) and approved in 2008 by the American Association for the Study of Liver Disease [2]. According to this scoring system, 25 WD patients (53.19\%) presented with a $47 \%$ decrease of serum ceruloplasmin compared to the normal level (normal level $11.75 \mathrm{mg} / \mathrm{dL}$; mean $( \pm \mathrm{SD})$ level of patients $(7.84 \pm 1.96 \mathrm{~g} / \mathrm{L}$; range $4.7-11.20 \mathrm{~g} / \mathrm{L})$. All investigated WD patients were found to have the Kayser-Fleischer ring by slit lamp examination, and their serum "free" copper levels in all serum samples were $<1.6 \mu \mathrm{mol} / \mathrm{L}$ (Table 1 ).

WD patients were also classified according to the main organs involved [1]. According to this classification, $19 \mathrm{WD}$ patients $(40.42 \%)$ had neurological presentation associated with symptomatic liver disease (N1, hepatocerebral form) and 28 patients (59.58\%) had neurological presentation not associated with symptomatic liver disease (N2, cerebral form). In the hepatocerebral group, one woman had a history 
Table 1 Demographic features of Ukrainian patients with Wilson's disease

\begin{tabular}{|c|c|c|c|}
\hline $\begin{array}{l}\text { Demographic features of } \\
\text { patients }\end{array}$ & $\begin{array}{l}\text { Cerebral form of WD } \\
(n=28)\end{array}$ & $\begin{array}{l}\text { Hepatocerebral form of WD } \\
(n=19)\end{array}$ & $\begin{array}{l}\text { All WD patients } \\
(n=47)\end{array}$ \\
\hline Number of patients, $n(\%)$ & $28(59.58 \%)$ & $19(40.42 \%)$ & $47(100 \%)$ \\
\hline Male, $n(\%)$ & $16(57.14 \%)$ & $8(42.1 \%)$ & $24(51.06 \%)$ \\
\hline Female, $n(\%)$ & $12(42.86 \%)$ & $11(57.9 \%)$ & $23(48.94 \%)$ \\
\hline Mean patient age (years) & $35.21 \pm 5.98(26-54)$ & $22.79 \pm 2.76(19-28)$ & $\begin{array}{l}30.19 \pm 7.87 \\
(19-54)\end{array}$ \\
\hline Mean WD duration (years) & $10.75 \pm 4.38(2-19)$ & $9.05 \pm 2.86(4-14)$ & $10.06 \pm 3.9(2-19)$ \\
\hline Mean age at WD onset (years) & $24.68 \pm 5.87(12-39)$ & $13.74 \pm 2.423(9-18)$ & $20.26 \pm 7.21(9-39)$ \\
\hline $\begin{array}{l}\text { Mean serum ceruloplasmin level } \\
(\mathrm{mg} / \mathrm{dL})\end{array}$ & $11.72 \pm 3.877(4.7-18.4)$ & $10.07 \pm 3.794(5.4-19.5)$ & $\begin{array}{c}11.05 \pm 3.89 \\
(4.7-19.5)\end{array}$ \\
\hline $\begin{array}{l}\text { Mean serum copper level } \\
(\mu \mathrm{mol} / \mathrm{L})\end{array}$ & $0.95 \pm 0.41(0.22-1.60)$ & $0.99 \pm 0.38(0.27-1.40)$ & $\begin{array}{l}0.97 \pm 0.4 \\
\quad(0.22-1.60)\end{array}$ \\
\hline
\end{tabular}

Values in table are presented as the mean \pm standard deviation (SD) with the range in parenthesis, unless indicated otherwise

$S D$ standard-deviation values, $W D$ Wilson's disease

of ovarian cyst (5.26\%), and two patients had complex partial seizures (10.53\%); in addition, brain injury was noted in the anamnesis of five patients $(26.31 \%)$. The demographic features of the investigated patients are presented in the Table 1.

\section{Tau Protein}

The tau levels in the serum samples collected from the WD patients were elevated compared to those of the HC (Fig. $1 ; p<0.0001, t$ statistic $6.047,95 \%$ confidence interval [CI] -218.2 to -95.86$)$. In the patient group, median total tau level (mean $\pm \mathrm{SD}$ ) was $221.7 \pm 135.1 \mathrm{pg} / \mathrm{mL}$ (lower to upper 95\% CI of mean 182.1-261.4); in the controls, it was $71.14 \pm 20.56 \mathrm{pg} / \mathrm{mL}$ (lower to upper 95\% CI of mean 63.47-78.82). Among the patients, 14 (29.78\%) were found to have a $34 \%$ increase in serum tau level (mean $221.7 \mathrm{pg} / \mathrm{mL}$ ) (Table 2).

To evaluate the clinical prognostic value of tau data, we further distinguished the different WD forms, i.e. the cerebral and hepatocerebral forms. The mean serum tau protein level was $231.3 \pm 31.1 \mathrm{pg} / \mathrm{mL}$ in patients with the cerebral form (mean age $35.21 \pm 5.98$ years), whereas it was $207.6 \pm 17.24 \mathrm{pg} / \mathrm{mL}$ in patients with the hepatocerebral form (mean age $22.79 \pm 2.76$ years). Thus, higher tau levels were observed in patients with the cerebral form than in those with the hepatocerebral form (Table 2); however, the difference between these two groups was not statistically significant (95\% CI -105.1 to $57.77, p>0.05$, $t$ statistic 0.5856 ).

Because the patients and controls were not gender matched, serum tau levels were compared between men and women (in HC and patients). The levels were not found to be comparable between male and female patients with WD $(p=0.168)$ and controls. In the same subset of samples, tau data were not correlated with age, age at disease onset and serum ceruloplasmin level ( $p=0.51$ for age, $p=0.63$ for age at disease onset). Although patients in the N2 group were older, disease duration did not differ to any great extent between groups. Our results showed no significant relationships between tau protein levels and copper dyshomeostasis; in all patients the serum copper levels remained low during this time (mean $0.97 \pm 0.4 \mu \mathrm{mol} / \mathrm{L})$, with a decrease in serum 


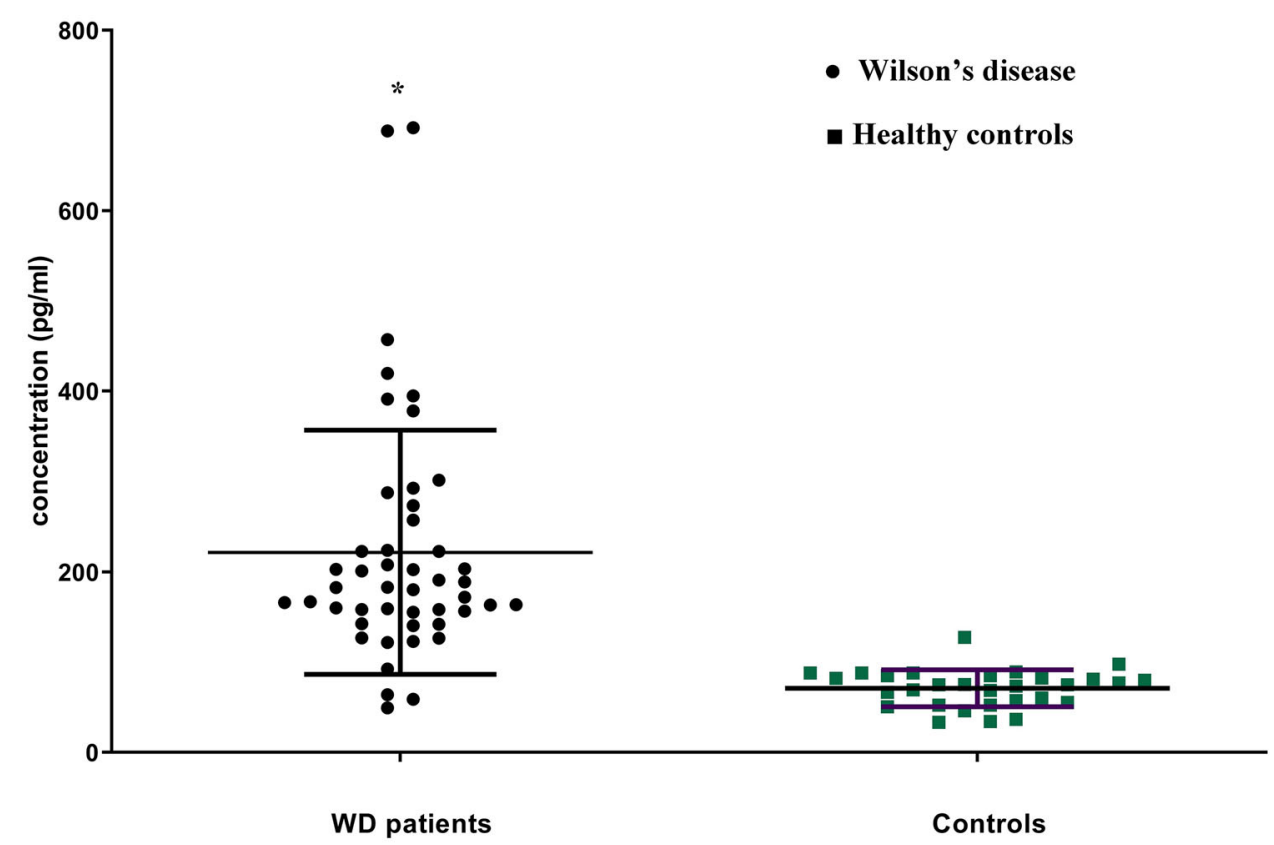

Fig. 1 Individual values of serum tau protein concentration in patients with Wilson's disease (WD) and healthy controls. Asterisk indicates that the difference between the patient group $(n=47)$ and healthy controls $(n=30)$ at baseline is significant at $p<0.0001$. Thick black horizontal line indicates the mean, whiskers indicate the range

Table 2 Tau protein levels in patients with Wilson's disease and healthy controls at baseline

\begin{tabular}{llllr}
\hline Defined parameter & $\begin{array}{l}\text { Cerebral form of WD } \\
(\boldsymbol{n}=\mathbf{2 8})\end{array}$ & $\begin{array}{l}\text { Hepatocerebral form of } \\
\text { WD }(\boldsymbol{n}=\mathbf{1 9})\end{array}$ & $\begin{array}{l}\text { All WD patients } \\
(\boldsymbol{n}=\mathbf{4 7})\end{array}$ & $\begin{array}{l}\text { Healthy controls } \\
(\boldsymbol{n}=\mathbf{3 0})\end{array}$ \\
\hline Tau protein serum & $231.3 \pm 31.1$ & $207.6 \pm 17.24$ & $221.7 \pm 135.1^{*}$ & $71.14 \pm 20.56$ \\
level $(\mathrm{pg} / \mathrm{mL})$ & $(49.5-692.2)$ & $(142.7-457.2)$ & $(49.5-692.2)$ & $(33.5-127.4)$ \\
\hline
\end{tabular}

Values in table are presented as the mean $\pm \mathrm{SD}$, with the range in parenthesis

${ }^{*} p<0.001$, indicating values are statistically significantly different vs. healthy controls

copper level of $31 \%$ compared to the normal value in 20 patients (42.55\%).

Patients with WD had abnormally high serum tau protein levels, with a tendency for patients with the late-onset cerebral WD form to have even higher tau levels. This increase was associated with baseline copper dyshomeostasis.

\section{DISCUSSION}

In this cross-sectional study, we demonstrated that serum tau protein levels were elevated in patients with WD, indicating axonal impairment and suggesting that tau protein may be involved in the pathogenesis of tau-mediated neurodegenerative processes in WD.

Tau protein is a highly soluble phosphoprotein and the most important protein of the axonal cytoskeleton $[6,10]$. This phosphoprotein mainly presents in the axon microtubules where a high degree of phosphorylation decreases its ability to promote and modulate microtubule stability and axon morphology [8]. Tau regulates microtubule dynamics, axonal transport and neurite outgrowth and might be 
involved in the establishment and maintenance of neuronal polarity $[11,29]$.

The axonal-derived phosphor-tau protein is significantly involved in multiple neurotoxic-degenerative mechanisms [12, 13, 30, 31]. In humans, tau hyperphosphorylation results in the formation of neurofibrillary tangles and neurophil threads, which are involved in the pathogenesis of AD and tauopathies. Neurofibrillary tangles associated with $\mathrm{AD}$ are composed mainly of paired helical filaments that result from the aggregation of abnormally phosphorylated tau protein. Some researchers have also reported the deposition of aggregates of different tau isoforms in other neurodegenerative diseases[29, 32].

Human studies have demonstrated that high levels of total tau in the cerebrospinal fluid (CSF) are frequently present not only in patients with neurodegenerative diseases, including $\mathrm{AD}$ $[9,11,12,30]$ and tauopathies $[5,13]$, but also in patients with brain trauma [15], acute ischemic stroke [20, 21], viral encephalitis $[4,17]$ and severe epileptic seizures [16], suggesting that tau protein levels in the CSF can reflect axonal brain damage. Other studies have shown high tau levels in patients with cerebral infections [4]. Thus, there is a well-established compilation of tau protein data from numerous clinical and animal studies, but to our knowledge the present study is the first large-scale study to investigate serum tau protein levels in WD patients, with a special focus on the relationship between different clinical forms, age at disease onset and ceruloplasmin and copper levels.

Copper is one of the main biometals, and it plays diverse roles in many vital important processes, including enzyme catalysis, protein stabilization and energy production [1-3, 22]. Previous studies have shown that impaired copper homeostasis and transition can enhance amyloid aggregation and neurotoxicity and trigger neurodegeneration $[2,3,25]$. The authors of recent studies [22-24] have proposed that copper could affect the accumulation of $\alpha$-synuclein, a protein involved in the pathogenesis of neuronal death [26]. Mechanisms involving the accumulation of copper to toxic levels may lead to widespread neurodegeneration as free copper is very toxic on its own and can cause irreversible cellular damage. Various mechanisms for impaired copper metabolism, copper intoxication and severe neurodegeneration have been proposed based on experimental findings; these include direct oxidative stress, unregulated apoptosis and loss of caspase-3 inhibitory control $[1,9,22,24]$. Published studies have shown that toxic copper deposits may induce oxidative stress, modify gene expression and enzyme catalysis and directly inhibit proteins and mitochondria function. In the brain, copper accumulates in astrocytes, leading to impairment of the blood-brain barrier and consequent neuron damage $[2,9,23,27,33]$.

The results of this study demonstrate that the mean $( \pm S D)$ level of tau protein increased in patients with WD as compared to the HC $(221.7 \pm 135.1$ vs. $\quad 71.14 \pm 20.56 \mathrm{pg} / \mathrm{mL}$, respectively), indicating axonal impairment in WD patients and revealing tau-mediated toxicity in WD that might be sufficiently pronounced as to be a potential cause or effect of neurodegeneration. This finding has implications for clinical management as tau level may be a good diagnostic tool to evaluate ongoing axonal damage associated with neurodegenerative processes during life such that tau protein may be an early marker of peripheral axonal damage. Tau protein has become the focus of research in studies on WD diagnostics as serum tau can be easily obtained, thereby providing the means to quantify early axonal injury.

We analyzed the prognostic value of tau protein as a biological marker of axonal damage and tau-mediated neurodegeneration in the 28 patients with the cerebral form and in the 19 patients with the hepatocerebral form who have had this disease for a mean of about 10 years. We report here that the investigated patients with WD had abnormally high levels of tau protein in both the cerebral and hepatocerebral clinical forms.

Tau is a new biomarker of interest in WD. Elevated serum tau protein levels have been associated with the presence of the late-onset cerebral clinical WD form, with median baseline serum tau levels tending to be higher in patients with the cerebral form of WD 
( $p=0.5611)$. This result suggests that tau protein may serve as a prognostic biomarker for cerebral forms of WD. Our two study groups (patients and HC) did not appreciably differ in terms of disease duration, age of disease onset and serum ceruloplasmin levels; also, men and women showed no differences in tau concentrations. The elevated serum levels of tauphosphor protein seems to reflect the destruction of neurons in the brain associated with baseline copper dyshomeostasis that was observed in all WD cases. Very high tau concentrations were found in $29.78 \%$ patients with WD, with all patients with WD being on a treatment course consisting of a stable dose of penicillamine.

WD is a disease leading to the progressive cell death of neurons and reactive astrogliosis where tau partly reflects WD neurodegeneration pathology [1-3]. Tau protein can be secreted across a damaged blood-brain barrier (BBB) and released into systemic circulation after the disintegration of neuron cells [34]. If the axonal integrity is interrupted for any reason, tau protein passes into the extracellular space and then to the CSF. Two pathophysiological mechanisms that cause this axonal loss have been proposed in the literature: inflammation and degeneration. According to this hypothesis, the source of tau protein in the CSF and blood is the transected axons $[4,12]$. The increase in CSF tau concentration, found not only in numerous neurodegenerative diseases but also in patients with MS [18-20] and brain trauma [16], support this hypothesis. One recent study, conducted on patients with $\mathrm{AD}$, suggests that tau protein may be released extracellularly by an exosomebased mechanism [32].

We can conclude that tau itself is the prominent agent implicated in the pathogenesis of many neurodegenerative diseases with axonal degeneration, but the key question on whether the elevation of serum tau protein level could reflect the primary disease pathogenesis processes or whether it occurs secondary to neurodegeneration in WD remains to be assessed in future studies. Tau-related neurodegeneration plays a specific role in our current understanding of the neurochemistry and pathogenesis of $\mathrm{WD}$, and the mechanisms underlying these interactions are largely unknown.

In the brain, copper accumulates predominantly in astrocytes, leading to BBB impairment with consequent damage to neurons and oligodendrocytes; the basal ganglia and brainstem are the regions with the highest susceptibility to copper toxicity. The increased level of free copper in the brain may also originate from the copper mobilized from brain parenchyma cells $[35,36]$, and not only from the serum. The copper-related toxic effects on the brain and BBB will lead to a worsening of the neurological conditions, which may be reflected in secondary neurodegeneration, which was measured here for the first time using the parameter of tau protein level. On the other hand, we cannot deny that tau protein level could reflect the pathological processes of the primary disease. However, the exact molecular mechanisms underlying abnormal copper metabolism, neurodegeneration, and tau protein levels are still insufficiently understood. The clinical presentation of WD can vary widely; consequently, the diagnosis of WD is not always straightforward [37]. Key WD features are liver disease and neuropsychiatric disturbances, and the diagnosis is especially difficult when WD presents with liver pathology, as none of the available laboratory tests is perfect. Gaining a better understanding of the molecular mechanism underlying the development of WD is imperative. Increased tissue copper levels may induce a series of harmful biochemical reactions, particularly oxidative stress, which can damage the structure and integrity of mitochondria, leading to neuron injury $[3,22,25]$. A better understanding of the exact molecular mechanisms causing aberrant copper deposition and of the mechanisms of tau-induced brain pathology are keys to developing effective management approaches for neurodegeneration where early detection of axonal impairment and early intervention are important steps in the prevention of disease progression.

Based on our results, we suggest that while elevated tau protein levels could be one of the causes of tau-mediated neurodegeneration processes in WD, they are most likely a result of neurodegenerative processes related to disease 
outcome. However, all patients in our study were on pharmacotherapy and it is more likely that copper can cause the release of tau into the circulation where the elevated tau level should be a mirror of neuronal damage. Our results also support the role of tau level as a prognostic factor in patients with the cerebral form of WD. We found that serum tau protein levels were increased in patients with WD and that tau levels tended to be higher in patients with the cerebral form. Taken together, our findings increase our knowledge of WD pathogenesis in humans.

\section{Limitations}

There are a number of inherent limitations to this study. The main limitation is the lack of objective measures of cognitive functions and neuroimaging abnormalities, such as the extent of atrophy rates (either hippocampal or whole brain) and contrast enhancing pattern to determine BBB breakage. However, we believe that it is extremely difficult to obtain these measures when examining patients with severe WD. The exact serum tau profile [38] in relation to cognitive dysfunction remains to be further assessed; such results will provide simple noninvasive tests to be useful in the stratification of the disease clinical form. The relatively small number of patients in this study limits us to conclude that tau protein may have a useful role in the monitoring of disease progression. Prospective investigations in this area should be performed.

\section{CONCLUSION}

The study provides novel data revealing high tau protein levels in patients with WD to be a indicator of axonal impairment. Consequently, serum tau protein could be as a potential biomarker for neuronal damage and may play a specific role in tau-mediated neurodegeneration contributing to WD pathogenesis.

\section{ACKNOWLEDGEMENTS}

Yevgeniya Lekomtseva would like to thank G.I. Gubina-Vakulik (Kharkiv National Medical University, Ukraine) for her great support in this research. The authors wish to thank the patients who participated in this study, which allowed this research to be possible.

Funding. No funding or sponsorship was received for this study or publication of this article.

Authorship. All named authors meet the International Committee of Medical Journal Editors (ICMJE) criteria for authorship for this manuscript, take responsibility for the integrity of the work as a whole, and have given final approval for the version to be published.

Disclosures. Yevgeniya Lekomtseva, Ivan Voloshyn-Gaponov and Gorbach Tatayna declare that they have nothing to disclose.

Compliance with Ethics Guidelines. All procedures performed in this study involving human participants were in accordance with local ethic committee and with the 1964 Helsinki declaration and its later amendments or comparable ethical standards. Informed consent was obtained from all individual participants included in the study.

Data Availability. The datasets used and/or analyzed during the current study are available from the corresponding author upon reasonable request.

Open Access. This article is distributed under the terms of the Creative Commons Attribution-NonCommercial 4.0 International License (http://creativecommons.org/licenses/ by-nc/4.0/), which permits any noncommercial use, distribution, and reproduction in any medium, provided you give appropriate credit to the original author(s) and the source, provide a link to the Creative Commons license, and indicate if changes were made. 


\section{REFERENCES}

1. Leggio L, Addolorato G, Abenavoli L, Gasbarrini G. Wilson's disease: clinical, genetic and pharmacological findings. Int J Immunopathol Pharmacol. 2005;18(1):7-14.

2. Kodama H, Fujisawa C, Bhadhprasit W. Inherited copper transport disorders: biochemical mechanisms, diagnosis, and treatment. Curr Drug Metab. 2012;13(3):237-50.

3. Pfeiffer RF. Wilson's disease. Semin Neurol. 2007;27(2):123-32.

4. Sussmuth SD, Reiber H, Tumani H. Tau protein in cerebrospinal fluid (CSF): a blood-CSF barrier related evaluation in patients with various neurological diseases. Neurosci Lett. 2001;300:95-8.

5. Uchihara T, Duyckaerts C, Seilhean D, Nakamura A, Lazarini F, Hauw JJ. Exclusive induction of tau2 epitope in microglia/macrophages in inflammatory lesions-tautwopathy distinct from degenerative tauopathies. Acta Neuropathol. 2005;109:159-64.

6. Chun J, Kwon T, Lee EJ, et al. 14-3-3 Protein mediates phosphorylation of microtubule-associated protein tau by serum- and glucocorticoid-induced protein kinase 1. Mol Cells. 2004;18(3):360-8.

7. Kadak MT, Cetin I, Tarakçıŏlu MC, Özer ÖF, Kaçar $S$, Çimen B. Low serum level $\alpha$-Synuclein and tau protein in autism spectrum disorder compared to controls. Neuropediatrics. 2015;46(6):410-5.

8. Daniels M. The role of microtubules in the growth and stabilization of nerve fibers. Ann Acad Sci. $1975 ; 253: 535-44$.

9. Jellinger KA. Recent advances in our understating of neurodegeneration. J Neural Transm (Vienna). 2009;116:1111-62.

10. Cleveland D, Hwo Y, Kirschner M. Purification of tau, a microtubule-associated protein that induces assembly of microtubules from purified tubulin. J Mol Biol. 1977;116:207-25.

11. Avila J, Lucas J, Perez M, Hernandez F. Role of tau protein in both physiological and pathological conditions. Physiol Rev. 2004;84:361-84.

12. Blennow K, Zetterberg H. Alzheimer disease. Lancet. 2006;368:387-403.

13. Delacourte A. Tauopathies: recent insights into old diseases. Folia Neuropathol. 2005;43:244-57.
14. Bulut M, Koksal O, Dogan S, et al. Tau protein as a serum marker of brain damage in mild traumatic brain injury: preliminary results. Adv Ther. 2006;23(1):12-22.

15. Wang J, Li J, Han L, et al. Serum $\tau$ protein as a potential biomarker in the assessment of traumatic brain injury. Exp Ther Med. 2016;11(3):1147-51.

16. Palmino J, Suhonen J, Keranen T, Hukkonen J, Peltola J, Pirttila T. Cerebrospinal fluid tau a marker of neuronal damage after epileptic seizure. Seizure. 2009;18:474-7.

17. Bartosik-Psuiek H, Stelmasiak Z. The CSF levels of total-tau and phosphor-tau in patients with relapsing-remitting multiple sclerosis. J Neural Transm (Vienna). 2006;113:339-45.

18. Kapaki E, Paraskevas GP, Michalopoulou M, Kilidireas $\mathrm{K}$. Increased cerebrospinal fluid tau protein in multiple sclerosis. Eur Neurol. 2000;43:228-32.

19. Terzi M, Birinci A, Cetinkaya E, Onar MK. Cerebrospinal fluid total tau protein levels in patients with multiple sclerosis. Acta Neurol Scand. 2007;115(5):325-30.

20. Hesse C, Rosengren L, Andreasen N, et al. Transient increase in total tau but not phosphor-tau in human cerebrospinal fluid after acute stroke. Neurosci Lett. 2001;297:187-90.

21. Lasek-Bal A, Jedrzejowska-Szypulka H, Rozycka J, Bal W, Kowalczyk A, Holecki M. The presence of Tau protein in blood as a potential prognostic factor in stroke patients. J Physiol Pharmacol. 2016;67(5):691-6.

22. Dusek P, Roos PM, Litwin T, Schneider SA, Flaten TP, Aaseth J. The neurotoxicity of iron, copper and manganese in Parkinson's and Wilson's diseases. J Trace Elem Med Biol. 2015;31:193-203.

23. Han $\mathrm{Y}$, Cheng $\mathrm{H}$, Toledo JB, et al. Impaired functional default mode network in patients with mild neurological Wilson's disease. Parkinsonism Relat Disord. 2016;30:46-51.

24. Koistinaho J, White AR, Kanninen KM. Biometals in rare neurodegenerative disorders of childhood. Front Aging Neurosci. 2013;25(5):14.

25. Rodriguez-Castro KI, Hevia-Urrutia FJ, Sturniolo GC. Wilson's disease: a review of what we have learned. World J Hepatol. 2015;7(29):2859-70.

26. Squitti R, Barbati G, Rossi L, et al. Excess of nonceruloplasmin serum copper in AD correlates with MMSE, CSF beta-amyloid, and h-tau. Neurology. 2006;11(67):76-82. 
27. Skowrońska M, Litwin T, Dzieżyc K, Wierzchowska A, Członkowska A. Does brain degeneration in Wilson disease involve not only copper but also iron accumulation? Neurol Neurochir Pol. 2013;47(6):542-6.

28. Zhang Y, Wang D, Wei W, Zeng X. Wilson's disease combined with systemic lupus erythematosus: a case report and literature review. BMC Neurol. 2018;18:85.

29. Roder HM, Hutton ML. Microtubule-associated protein tau as a therapeutic target in neurodegenerative disease. Expert Opin Ther Targets. 2007;11(4):435-42.

30. Gotz J, Gladbach A, Pennanen L, et al. Animal models reveal role for tau phosphorylation in human disease. Biochim Biophys Acta. 2010;1802(10):860.

31. Herukka SK, Hallikainen M, Soininen H, Pirttila T. CSF A 342 and tau or phosphorylated tau and prediction of progressive mild cognitive impairment. Neurology. 2005;64:1294-7.

32. Feany MB, Ksiezak-Reding H, Liu W, Vincent I, Yen SH, Dickson DW. Epitope expression and hyperphosphorylation of tau protein in corticobasal degeneration: differentiation from progressive supranuclear palsy. Acta Neuropathol. 1995;90:37-43.

33. Bandmann O, Weiss KH, Kaler SG. Wilson's disease and other neurological copper disorders. Lancet Neurol. 2015;14(1):103-13.

34. Takahashi K, Hasegawa S, Maeba S, et al. Serum tau protein level serves as a predictive factor for neurological prognosis in neonatal asphyxia. Brain Dev. 2014;36(8):670-5.

35. Chen DB, Feng L, Lin XP, Zhang W, Li FR, Liang XL, $\mathrm{Li}$ XH. Penicillamine increases free copper and enhances oxidative stress in the brain of toxic milk mice. PLoS One. 2012;7(5):e37709. https://doi.org/ 10.1371/journal.pone.0037709.

36. Scheiber IF, Bruha R, Dusek P. Pathogenesis of Wilson disease. Handb Clin Neurol. 2017;142: 43-55.

37. Wu F, Wang J, Pu C, Qiao L, Jiang C. Wilson's disease: a comprehensive review of the molecular mechanisms. Int J Mol Sci. 2015;16:6419-31.

38. Lue LF, Guerra A, Walker DG. Amyloid beta and tau as Alzheimer's disease blood biomarkers: promise from new technologies. Neurol Ther. 2017;6:S25-36. 